\title{
Chemotherapy Induced Cardiotoxicity: Facts, Breakthroughs, and Challenges
}

\author{
Wael Maharsy ${ }^{1}$ \\ ${ }^{1}$ Faculty of Medicine, University of Ottawa
}

A BSTRACT

Chemotherapy involves the use of one or more cytotoxic drugs to kill rapidly dividing malignant cells. One of the most promising fields of chemotherapy is the so-called targeted therapy, where a group of antibodies and small-molecule kinase inhibitors are designed to target key proteins involved in growth and proliferation pathways. Today, targeted therapeutics, such as Imatinib mesylate (Gleevec), have radically transformed the treatment of solid tumors and some blood malignancies. Unfortunately, emerging chemotherapy-associated cardiotoxicity poses unexpected challenges that may limit effective use of these novel drugs. Drug-induced cardiotoxicity is associated with cardiac cell death and cardiac dysfunction, which can lead to life threatening heart failure. The mechanisms underlying this cardiotoxicity remain poorly understood. In this review we discuss the cardiotoxicity of some of the major anticancer chemotherapy drugs, and summarize recent insights into the mechanisms implicated in cardiac cell death and survival.

\section{RÉ S U MÉ}

\section{THE CANCER AND ANTICANCER TREATMENTS}

Cancer is a malignant growth caused by the uncontrolled proliferation of abnormal cells. Cancerous cells often have genomic alterations at multiple sites producing mutations that, among others, activate oncogenes and repress tumor suppressor genes. Presently, more than a hundred different types of tumors targeting almost all body organs have been described [1,2]. Extensive research into tumor etiologies and cancer progression has resulted in a plethora of anticancer drugs and regimens with varying efficiency at eradicating the disease. At present, cancer therapies can involve surgery, chemotherapy, radiation therapy, and cellular therapy or a combination thereof [3]. This review focuses on chemotherapy and the associated cardiac toxicity often seen in cancer survivors.

Chemotherapy involves a group of cytotoxic drugs that kill rapidly dividing cells by interfering with cell division and DNA synthe- sis. The subsequent side effect of this approach is that chemotherapy also harms healthy, rapidly dividing cells, such as those lining the gut and forming the hair follicle. Chemotherapy, which is performed to either cure or control cancers, can be used alone or combined with radiation therapy or surgery [3]. A promising field of chemotherapy is the so-called 'targeted therapy', where antibodies or small-molecule inhibitors are designed to target key pro-tumorigenic growth inducing cell surface receptors and/ or components of their intracellular signaling pathways such as protein tyrosine kinases. Currently, more than 10 FDA-approved agents exist, with many more awaiting approval, offering hope to patients with cancers that are unresponsive to other treatment modalities. Interestingly, the high mutation rates affecting protein kinases in cancers, and their subsequent cancer-driving roles has triggered the search for kinase-specific inhibitory molecules. Today, targeted therapeutics such as Imatinib mesylate (Gleevec), a small molecule targeting the $B C R-A B L$ fusion protein kinase that causes chronic myelogenous leukemia $(\mathrm{CML})$, have drasti-

Keywords: Chemotherapy; Cardiotoxicity; Cancer; Cardiac Dysfunction 
cally transformed the treatment of this malignancy $[4,5]$. Other pathways, such as the Phospho-Inositol 3 Kinase (PI3K) pathway are also altered in cancer due to mutations or amplifications of several key components, thus making them attractive targets to drug development. Not surprisingly, the pharmaceutical industry is pursuing agents that can inhibit either receptor tyrosine kinases upstream of, or pro-growth kinases associated with the PI3K pathway. There are several agents already in clinical trials, such as the multikinase inhibitor Lenvatinib and the splenic tyrosine kinase inhibitor Fostamatinib [4]. Another aspect of cancer is the dysregulation of cell cycle regulators. Therefore, targeting these factors is currently a major focus in cancer research. In fact, numerous cyclin-D kinases (CDKs) inhibitors, which target these pro-proliferation kinases, are in development with many in clinical trials $[3,4,5]$.

\section{CARDIOTOXICITY OF CHEMOTHERAPEUTIC AGENTS}

Chemotherapy-induced cardiotoxicity is a serious clinical problem faced by both cardiologists and oncologists (Table 1). Contractile heart cells, known as cardiomyocytes, are prone to short-term or permanent injury upon exposure to toxic agents such as recreational drugs and therapeutic agents. Unlike most other cells, postnatal cardiomyocytes have limited regenerative capacity. Thus, their loss can cause cardiac dysfunction, which in turn can lead to heart failure, a disease with worse prognosis than some cancers [6]. The realization that chemotherapy might worsen an underlying cardiac problem or create a new one did not become a concern for cardiologists and oncologists until the seventies. The cardiac toxicity of anthracyclines, a class of bacterial antibiotics widely used in chemotherapy, was the first to be described [4-6]. This consequently spurred awareness and extensive research into basic and clinical aspects of chemotherapy associated cardiotoxicity. Although much of the literature focuses on causes and mechanisms implicated in anthracycline-induced cardiomyopathy, other types of chemotherapy-related cardiac toxicity are also common. For example, Cytarabine, an antimetabolic agent, can negatively affect the cardiac vasculature and the pericardium resulting in ischemia and changes in blood pressure, as well as an imbalance in liquid equilibrium and pericardial thickening, respectively [7]. Finally, anticancer drugs such as Imatinib and anthracyclines can aggravate or induce cardiac arrhythmias and other cardiac conditions in patients $[4,8-10]$.

In anthracycline-induced cardiac myopathy, left ventricle (LV) systolic dysfunction is dose-dependent and damage is irreversible, eventually leading to heart failure. Anthracyclines and other drugs that cause irreversible cell destruction are known as type I agents $[6,8]$. In contrast, many novel drugs that also cause cardiomyopathies, like the monoclonal antibody trastuzumab used mostly in breast cancer treatment, or the VEGF receptor antibody Bevacizumab, usually induce reversible cardiac damage and are therefore referred to as type II agents. Type II agent-induced toxicity is not dose dependent and in most cases reversible upon drug withdrawal [8]. The cardiotoxicity of anthracyclines, and various targeted chemotherapeutic drugs will be the focus of the current review.

\section{ANTHRACYCLINES-INDUCED CARDIOTOXICITY}

Anthracyclines have been effectively used as anticancer agents over the past fifty years. They are used in the treatment of leukemias, lymphomas, breast cancer, and sarcomas. They induce cell death through mechanisms that involve DNA intercalation, topoisomerase II inhibition, as well as replication and transcription inhibition. At present, the use of anthracyclines is limited due to their negative effects on the heart, often resulting in cardiomyopathy [8].

Doxorubicin (DOX), the most commonly used anthracycline, has been used since the late 1960s. Tumors treated include esophageal and breast carcinomas, osteosarcoma, soft tissue sarcomas, Kaposi's sarcoma, and Hodgkin's and non-Hodgkin's lymphomas [10]. Furthermore, in many breast cancer patients, DOX is used in conjunction with trastuzumab, a monoclonal antibody against the oncogenic human epidermal growth factor receptor 2 (HER2, ErbB-2) [11]. As mentioned earlier, the use of DOX has been subdued by reports of fatal cardiotoxic events in cancer patients $[1,8,9]$. Mechanistically, DOX is known to intercalate DNA and inhibit replication, as well as form a complex with iron, increasing free radical production and inducing oxidative damage characterized by membrane disruption and cellular dysfunction $[8,9]$. Such effects are especially detrimental to cardiomyocytes, which are highly susceptible to oxidative damage. Other mechanisms that contribute to cardiotoxicity include mitochondrial DNA mutations, calcium handling alterations, and dysregulation of important cardiac transcription factors, such as GATA4 [9]. DOXinduced cardiac toxicity is dose-dependent and cellular damage ranging from vacuolation and contractile elements disarray to cell death, is irreversible $[6,8]$.

Latent DOX associated cardiomyopathies usually occur within one month to one year following treatment induction, but can be observed months after the end of treatment. Known risk factors for cardiotoxicity include older age, hypertension, diabetes, previous cardiac disease, and simultaneous treatment with other anticancer drugs [10]. Various strategies have been developed to diminish the risk of cardiac injury, like the use of DOX analogues, liposomal delivery systems, and the co-administration of putative cardioprotective agents. These approaches have had limited success with the most effective being dosage limitation and alternative drug delivery methods [8]. Treatments of the resulting cardiomyopathy include angiotensin-converting enzyme (ACE) inhibitors, diuretics, beta-blockers, and digoxin. DOX continues to be used in cancer therapy due to its efficacy in the treatment of many tumors $[8,10]$. 
Over the past decades, research on DOX associated cardiotoxicity failed to elucidate its underlying mechanism(s). However, most studies agree on the pivotal role played by DOX-induced oxidative stress, given that DOX chemical structure possesses an inherent tendency to generate free radicals and reactive oxygen species (ROS) during its metabolism. Moreover, mitochondrial DNA damage caused by DOX directly or by ROS was found to cause respiratory chain failure and increased ROS production [12]. On the other hand, DOX cardiotoxicity is enhanced by dysregulation of several pathways including calcium handling, the adrenergic system, and inhibition of critical cardiomyocyte-specific gene(s), such as transcription factor GATA-4. GATA-4, a major regulator of heart development and a postnatal myocytes' survival factor, is depleted rapidly in response to DOX treatment [6]. Interestingly, GATA-4 downregulation was associated with concomitant decrease in both antiapoptotic BCL-XL and BCL-2 gene expression, leading to increased apoptotic cell death. Conversely, enhancing GATA-4 activity by the $\alpha 1$ adrenergic agonist phenylephrine or the adenovirus-mediated overexpression of GATA-4, prevented DOX-induced apoptosis in cardiomyocytes mainly via the transcriptional activation of both BCL-XL and BCL-2 $[6,13]$.

Most of the cellular events mentioned above trigger cardiomyocyte death mainly via apoptosis and necrosis, but other cell death forms, like autophagy, might also play an important role $[12,13]$. DOX-induced oxidative stress and the subsequent effect on cytosolic calcium homeostasis leads to mitochondrial calcium overload, which in turn triggers mitochondrial permeability transition (MPT) [12]. MPT results in loss of membrane potential, swelling, and outer membrane rupture, releasing cytochrome c and initiating the intrinsic apoptosis pathway [12]. Other studies found that cardiomyocyte death in DOX-induced cardiac dysfunction can be explained partly through a Fas-mediated extrinsic apoptosis pathway [14]. Moreover, necrosis or the more recently described programmed necrosis could play a major role in DOXinduced cardiotoxicity. Mechanistically, ATP depletion caused by mitochondrial uncoupling could favor necrotic rather than apoptotic cell death [15]. Finally, DOX was also found to increase autophagic fluxes in cardiomyocytes essentially contributing to its cardiotoxicity. Once again, increased GATA-4, which controls many genes involved in mitochondrial biogenesis and stress response, inhibited DOX-induced autophagy and reduced cell death, reinstating the central role of GATA-4 as a cardioprotective, prosurvival signal in adult cardiomyocytes [6].

\section{MONOCLONAL ANTIBODIES AND ASSOCIATED CARDIOTOXICITY}

In the past decade, the use of monoclonal antibodies that can specifically block the action of oncogenic proteins, mostly growth receptors linked to tyrosine kinases, has significantly improved overall survival of patients suffering from certain cancers (Table 1). For example, trastuzumab (Herceptin) and pertuzumab, are monoclonal antibodies used to antagonize and block the on- cogenic activity of HER2 (ErbB2). HER2, which is upregulated in $25-30 \%$ of breast cancer patients (HER2+), is responsible for the development and progression of several types of aggressive breast cancers. The mutant, constitutively active HER2 receptor activates growth (MAPK) and proliferation (PI3K-AKT) pathways leading to the uncontrolled division of cancer cells. Trastuzumab and pertuzumab can bind the hyperactive HER2 receptor, forcing its internalization and degradation, and eventually causing cancer cell death [16]. These antibodies are thus potent and widely used breast cancer targeted chemotherapeutic agents, and are usually administered in conjunction with DOX for maximum anticancer efficiency and better patient prognosis [17]. The reported percentage of trastuzumab linked cardiotoxic events in patients ranges between $1.7 \%$ and $20 \%[17,18]$. The cardiotoxicity associated with anti-HER2 therapy appears to be dose independent and reversible, although there is present controversy regarding the reversible nature of cardiac damage. Mechanistically, ErbB2 blockade can induce cardiomyocyte cell death through the activation of the mitochondrial apoptotic pathway [19]. Previous or concomitant exposure to DOX increases the risk of cardiac events in anti-HER2 treated patients. Some of the suggested mechanisms include the downregulation of several prosurvival pathways (ERK and AKT pathways), increased accumulation of DOX in cardiomyocytes, and impaired angiogenic and myogenic cardiac programs [20].

Other monoclonal antibodies used as chemotherapeutic agents include the anti-angiogenic Bevacizumab, which binds to vascular endothelial growth factor (VEGF) and Ramucirumab, which binds to the type-2 receptor (VEGFR2). These antibodies potently suppress the proangiogenic VEGF/VEGFR pathway and thus limit angiogenesis in tumors. Unfortunately, the inhibition of the VEGF/VEGFR pathway triggers pathologic alterations in the cardiovascular system [21]. Clinically, around $10 \%$ of patients receiving bevacizumab or ramucirumab develop hypertension and $2-3 \%$ receiving bevacizumab suffer from heart failure $[22,23]$.

\section{KINASE INHIBITORS AND CARDIOTOXICITY}

Kinase inhibitors (KIs) are small molecules that mostly compete with ATP for binding to the kinase ATP pocket. The binding of these molecules blocks the phospho-transferase activity of an oncogenic kinase preventing the phosphorylation of downstream substrates. KIs bind the pocket with a very high affinity at low cellular concentrations ( $\mathrm{nM}$ to $\mu \mathrm{M}$ ), given the abundance of ATP in a cell (mM) [24]. Type I inhibitors, which target the ATP pocket only, are less selective and often lead to off-target effects and potential toxicity. This poor selectivity is addressed in type II inhibitors, like Imatinib mesylate, which usually recognize other regions of the kinase in addition to the ATP pocket. Type II inhibitors are thus more selective and typically more potent because they can bind and inhibit the kinase in both its active and inactive conformations. Finally, type III inhibitors, target kinase-specific non-conserved regions different than the ATP pocket, and there- 
fore, are excellent selective KIs. Unfortunately, type III inhibitors represent a very small percentage of Kls due largely to designassociated challenges. Interestingly, the superior effectiveness of more selective inhibitors over non-selective ones has been challenged in a variety of oncological and inflammatory diseases, given that non-selective KIs can target other kinases that might be essential for disease progression and would typically lead to a better anticancer efficacy [24]. Finally, non-selective KIs can be used in more cancers and may be more appealing for drug development.

The human kenome is composed of 518 kinases, out of which around 90 are tyrosine kinases (TKs) [25]. Tyrosine kinases can be either receptor tyrosine kinases (RTKs) or non-receptor TKs. TKs are usually heavily mutated or amplified in cancers and therefore the development of therapeutic TK inhibitors (TKIs) has exploded recently. Imatinib mesylate (Gleevec, Novartis), was the first TKI to reach market following FDA approval in 2001. Imatinib approval as an anticancer drug paved the way for several tyrosine-kinase-targeted therapies [25]. Imatinib inhibits the tyrosine kinase activity of the BCR-ABL fusion protein resulting from a chromosomal translocation, known as the Philadelphia chromosome. The BCR-ABL fusion protein dimerization phosphorylates the $A B L$ kinase domain leading to its constitutive activation. This constitutively active kinase activates antiapoptotic pathways (RAS-ERK, PI3K-AKT, JAK-STAT), ultimately enhancing cell division and inhibiting DNA repair leading to chronic myelogenous leukemia (CML) [Reviewed in 26]. During the pre-Imatinib era, CML was treated by anthracyclines, interferons, and bone marrow transplantation albeit with variable successes [reviewed in 27]. The discovery of Imatinib revolutionized the treatment of CML patients, with over $70 \%$ cytogenic remission and initially reported minimal side effects, which included peripheral edema and dyspnea. In addition to its inhibition of BCR-ABL, Imatinib inhibits the receptor for stem cell factor (c-KIT), usually upregulated in gastrointestinal stromal tumors (GISTs), and platelet derived growth factor receptors (PDGFRs), which play important roles in other cancers including GIST and glioblastoma [24]. Lately, Imatinib use has been extended to the treatment of more than 10 solid cancers including gastrointestinal stromal tumors and prostate cancer [Reviewed in 26].

Numerous reports on the effect of Imatinib therapy on the development of congestive heart failure (CHF) emerged recently [28-30]. These studies have been highly controversial given the results of IRIS (International Randomized Study of Interferon versus STI571, Imatinib), where the overall incidence of CHF was about $1 \%$ in both the imatinib and the interferon arms [31-33]. The discrepancy could be attributed to the lack of accurate and extensive cardiac function monitoring in most cancer clinical trials, and/or to variables that can affect cardiovascular parameters like age, sex, or obesity. When tested in vitro in cultured cardiomyocytes and in vivo in mice, Imatinib was found to induce cell death associated with early apoptotic features, and to lead to compromised cardiac function, proving that it has the potential to cause CHF. Our lab as well as others found that imatinib-induced cardiomyocyte death was triggered or at least associated with a dysregulated mitochondrial function [26,34], an anomaly aggravated in aging hearts [35].

Mechanistically, Imatinib-induced cardiotoxicity was suggested to be partly due to its direct inhibition of c-ABL in cardiomyocytes. Furthermore, the endoplasmic reticulum stress response was found to be activated in treated cardiac myocytes [34], a situation known to ultimately provoke cell death. Although the pathways implicated in Imatinib-induced cardiac toxicity are still not fully understood, we recently reported that the upregulation of $\mathrm{Bcl}-2$, an antiapoptotic protein, protects against imatinib cardiotoxicity. Remarkably, we also found that this cardiotoxicity is age-dependent and more evident in old mice. Our results provided new insights into the mechanism of Imatinib negative effects on the heart and offered a possible explanation for the current controversy regarding imatinib-induced cardiotoxicity in cancer patients [26]. Interestingly, a new study found that chronic imatinib treatment induces intracellular calcium ion accumulation, pathologic hypertrophy and remodeling, as well as cell death in ex vivo and in vivo cardiac models [36].

Sunitinib, which is used against renal cell carcinoma and gastro intestinal stromal tumors, and inhibits VEGFRs, PDGFR, and c-kit, was another multitargeted TKI shown to induce cardiotoxicity in cancer patients. Chu et al. reported that $28 \%$ of patients receiving the approved dose had $10-15 \%$ reductions in the left ventricular ejection fraction (LVEF). They also found that sunitinib induced an increase in mean blood pressure and around $47 \%$ of patients developed hypertension [37]. Sunitinib induced mitochondrial injury and apoptosis in cultured rat cardiomyocytes and mice [37]. Finally, in an in vitro toxicity assay, sunitinib triggered cardiomyocyte death, metabolic abnormalities, and lipid accumulation. In this same assay, other TKIs were tested and each showed a distinct toxicity profile reflecting the complex and multiple mechanisms implicated in this drug-induced cardiotoxicity [38]. This lack of target specificity and multiple off target inhibition hypothesis is believed to be the major contributor to cardiomyocyte damage and the wide array of cardiovascular anomalies associated with small molecule kinase inhibitors. Table 1 lists various kinase inhibitors, their target kinases, treated malignancies, and associated cardiovascular events.

\section{DISCUSSION}

Cardiac toxicity associated with traditional and targeted chemotherapy has led to the emergence of the new field of cardiac oncology. Lately, many hospitals and health centres have established on-site specialized cardiac oncology units, where oncologists and cardiologists jointly assess individual clinical cases in order to pre-emptively develop carefully monitored treatment protocols. Basic and translational research done on the increas- 
ing number of novel antineoplastic drugs has had a huge impact, and scientific breakthroughs continue to shape cancer care protocols in Canada and the rest of the world. Unfortunately, poorly understood roles of many kinases in the heart, as well as the multi-target specificity of small molecule inhibitors are still major limitations facing researchers and health practitioners. On the bright side, with better awareness and development of early biomarkers, such as plasma BNP, cardiotoxicity might become a manageable clinical challenge, but its prevention requires further insight into cardiomyocyte survival, and the development of appropriate drugs targeting these pathways.

\section{CONCLUSION}

Understanding cardiac survival pathways would ultimately lead to the development of heart-friendly chemotherapy regimens, or at least cardioprotective designer molecules that would prevent/ counterbalance the adverse effects of anticancer drugs on the heart. This important and complex undertaking requires interdisciplinary collaboration among medicinal chemists, biomedical researchers, and clinical scientists from oncology and cardiology. Eradicating chemotherapy induced cardiac toxicity is a formidable challenge to translational medicine.

\section{ACKNOWLEDGEMENTS}

Work in our laboratory is supported by the Canadian Institute of Health Research and The Heart and Stroke Foundation of Canada.

\section{REFERENCES}

1. Umar A, Dunn BK, Greenwald P. Future directions in cancer prevention. Nature Reviews Cancer. 2012; 835-848.

2. National Cancer Institute. What is cancer? 2015. [updated 2015 Feb 9; cited 2015 March 30]. Available from: www.cancer.gov/cancertopics/What-iscancer.

3. Walther V, Hiley CT, Shibata D, et al. Can oncology recapitulate paleontology? Lessons from species extinctions. Nature Reviews Clinical Oncology. 2015; 1-13.

4. Cheng $\mathrm{H}$, Force $\mathrm{T}$. Why do kinase inhibitors cause cardiotoxicity and what can be done about it? Progress in Cardiovascular Diseases. 2010; 53(2):114120.

5. Vivanco I. Targeting molecular addictions in cancer. British Journal of Cancer. 2014; 111(11):2033-2038.

6. Aries A, Paradis P, Lefebvre C, Schwartz RJ, Nemer M. Essential role of GATA4 in cell survival and drug-induced cardiotoxicity. Proceedings of the National Academy of Sciences. 2004; 101(18):6975-6980.

7. Conrad ME. Cytarabine and Cardiac Failure. American Journal of Hematology. 1992; 41(2):143-144.

8. Ewer MS, Ewer SM. Cardiotoxicity of anticancer treatments: What the cardiologist needs to know. Nature Reviews. Cardiology. 2010; 7(10):564-575.

9. Geiger S, Lange V, Suhl P, Heinemann V, Stemmler H-J. Anticancer therapy induced cardiotoxicity: Review of the literature. Anti-cancer Drugs. 2010; 21(6):578-590.

10. Figueredo VM. Chemical cardiomyopathies: The negative effects of medications and nonprescribed drugs on the heart. The American Journal of Medicine. 2011; 124(6):480-488.

11. Zeglinski M, Ludke A, Jassal DS, Singal PK. Trastuzumab-induced cardiac dysfunction: A "dual-hit". Experimental and Clinical Cardiology. 2011; 16(3):7074.

12. $H$, Chen $X, G a o ~ E$, et al. Increasing cardiac contractility after myocardial infarction exacerbates cardiac injury and pump dysfunction. Circulation Research. 2010; 107(6):800-809.
13. Kobayashi S, Lackey $\mathrm{T}$, Huang $\mathrm{Y}$, et al. Transcription factor GATA4 regulates cardiac BCL2 gene expression in vitro and in vivo. FASEB. 2006; 20(6):800802.

14. Niu J, Azfer A, Wang K, Wang X, Kolattukudy PE. Cardiac-targeted expression of soluble Fas attenuates doxorubicin-induced cardiotoxicity in mice. Pharmacology. 2009; 328(3):740-748.

15. Whelan RS1, Konstantinidis K, Wei AC, et al. Bax regulates primary necrosis through mitochondrial dynamics. Proceedings of the National Academy of Sciences. 2012; 109(17):6566-6571.

16. Hudis, C. A. Trastuzumab--mechanism of action and use in clinical practice. New England Journal of Medicine. 2007; 357(1):39-51.

17. Bang YJ, Van Cutsem E, Feyereislova A, et al. Trastuzumab in combination with chemotherapy versus chemotherapy alone for treatment of HER2-positive advanced gastric or gastro-oesophageal junction cancer (ToGA): a phase 3, open-label, randomised controlled trial. Lancet. 2010; 376(9749):687-697.

18. Smith KL, Dang C, Seidman AD. Cardiac dysfunction associated with trastuzumab. Expert Opinion on Drug Safety. 2006; 5:619-629.

19. Gordon LI, Burke MA, Singh AT, et al. Blockade of the erbB2 receptor induces cardiomyocyte death through mitochondrial and reactive oxygen speciesdependent pathways. Journal of Biological Chemistry. 2009; 284(4):2080 2087.

20. Milano G, Raucci A, Scopece A, et al. Doxorubicin and trastuzumab regimen induces biventricular failure in mice. Journal of the American Society of Echocardiography. 2014; 27(5):568-579.

21. Scott JM, Lakoski S, Mackey JR, et al. The potential role of aerobic exercise to modulate cardiotoxicity of molecularly targeted cancer therapeutics. The Oncologist. 2013; 18(2):221-231.

22. Miller K, Wang M, Gralow J, et al. Paclitaxel plus bevacizumab versus paclitaxel alone for metastatic breast cancer. New England Journal of Medicine. 2007; 357(26):2666-2676.

23. Spratlin JL, Mulder KE, Mackey JR. Ramucirumab (IMC-1121B): a novel attack on angiogenesis. Future Oncology. 2010; 6(7):1085-1094.

24. Force T. Introduction to cardiotoxicity review series. Circulation Research. 2010; 106(1):19-20.

25. Force T, Krause DS, Van Etten RA. Molecular mechanisms of cardiotoxicity of tyrosine kinase inhibition. Nature Reviews. Cancer. 2007; 7(5):332-344.

26. Maharsy W, Aries A, Mansour O, Komati H, Nemer M. Ageing is a risk factor in imatinib mesylate cardiotoxicity. European Journal of Heart Failure. 2014; 16(4):367-376.

27. Mcglave PB, Shu XO, Wen W, et al. Unrelated donor marrow transplantation for chronic myelogenous leukemia: 9 years experience of the National Marrow Donor Program. Hematology. 2000; 95:2219-2225.

28. Demetri GD. Structural reengineering of imatinib to decrease cardiac risk in cancer therapy. Cancer. 2007; 117(12):2005-2008.

29. Turrisi G, Montagnani F, Grotti S, Marinozzi C, Bolognese L, Fiorentini G. Congestive heart failure during imatinib mesylate treatment. International Journal of Cardiology. 2010; 145(1):148-150.

30. Toubert ME, Vercellino L, Faugeron I, Lussato D, Hindie E, Bousquet G. Fatal heart failure after a 26-month combination of tyrosine kinase inhibitors in a papillary thyroid cancer. Thyroid; $21(4): 451-454$.

31. O'Brien SG, Meinhardt P, Bond E, et al. Effects of imatinib mesylate (STI571, Glivec) on the pharmacokinetics of simvastatin, a cytochrome p450 3A4 substrate, in patients with chronic myeloid leukaemia. British Journal of Cancer. 2003; 89(10):1855-1859.

32. Trent JC, Patel SS, Zhang J, et al. Rare incidence of congestive heart failure in gastrointestinal stromal tumor and other sarcoma patients receiving imatinib mesylate. Cancer. 2010; 116(1):184-192.

33. Wolf $A$, Couttet $P$, Dong $M$, et al. Imatinib does not induce cardiotoxicity at clinically relevant concentrations in preclinical studies. Leukemia Research. 2010; 34(9):1180-1188.

34. Kerkelä R, Grazette L, Yacobi R, et al. Cardiotoxicity of the cancer therapeutic agent imatinib mesylate. Nature Medicine. 2006; 12(8):908-916.

35. Raju R, Jian B, Hubbard W, Chaudry I. The mitoscriptome in aging and disease. Aging and Disease. 2011; 19;2(2):174-180

36. Barr LA, Makarewich CA, Berretta RM, et al. Imatinib activates pathological hypertrophy by altering myocyte calcium regulation. Clinical and Translational Science. 2014; 7(5):360-367.

37. Chu TF, Rupnick MA, Kerkela R, et al. Cardiotoxicity associated with tyrosine kinase inhibitor sunitinib. Lancet. 2007; 370(9604):2011-2019.

38. Doherty KR, Wappel RL, Talbert DR, et al. Multi-parameter in vitro toxicity testing of crizotinib, sunitinib, erlotinib, and nilotinib in human cardiomyo- 
cytes. Toxicology and Applied Pharmacology. 2013; 272(1):245-55.

39. Portera CC, Walshe JM, Rosing DR, et al. Cardiac toxicity and efficacy of trastuzumab combined with pertuzumab in patients with human epidermal growth factor receptor 2-positive metastatic breast cancer. Clinical Cancer Research. 2008; 14(11):2710-2716.

40. Sendur MA, Aksoy S, Altundag K. Cardiotoxicity of novel HER2-targeted therapies. Current Medical Research and Opinion. 2013; 29(8):1015-1024.

41. Orphanos GS, loannidis GN, Ardavanis AG. Cardiotoxicity induced by tyrosine kinase inhibitors. Acta Oncologica. 2009; 48(7):964-970.

Table 1. Targeted chemotherapeutic agents, targets, treated cancers, and associated cardiotoxicity.

\begin{tabular}{|c|c|c|c|c|}
\hline Agent & Class & Targets & Cancer & Cardiotoxicity \\
\hline Trastuzumab & $\mathrm{mAb}$ & HER2 & HER2+ breast cancer & $\begin{array}{l}\text { CHF, Asymptomatic } \\
\text { LVEF decline }[16,17]\end{array}$ \\
\hline Pertuzumab & $\mathrm{mAb}$ & HER2 & HER2+ breast cancer & $\begin{array}{l}\text { CHF, Asymptomatic } \\
\text { LVEF decline [38] }\end{array}$ \\
\hline Bevacizumab & $\mathrm{mAb}$ & VEGF & $\begin{array}{c}\text { RCC, CRC, glioblas- } \\
\text { toma, NSCLC, } \\
\text { breast cancer }\end{array}$ & $\begin{array}{c}\text { Asymptomatic LVEF } \\
\text { decline, Hypertension } \\
{[21,22]}\end{array}$ \\
\hline Imatinib & TKI & ABL, c-KIT, PDGFR & $\begin{array}{l}\text { CBL, GIST, } \\
\text { glioblastoma }\end{array}$ & $\begin{array}{c}\text { CHF, Asymptomatic } \\
\text { LVEF decline } \\
{[24,30,27-29,33]}\end{array}$ \\
\hline Sunitinib & TKI & $\begin{array}{c}\text { VEGFR, } \\
\text { c-KIT, PDGFR }\end{array}$ & GIST, RCC & $\begin{array}{c}\text { CHF, Asymptomatic } \\
\text { LVEF decline, Hyperten- } \\
\text { sion [36] }\end{array}$ \\
\hline Lapatinib & TKI & ErbB1, HER2 & $\begin{array}{l}\text { HER } 2+\text { breast cancer, } \\
\text { ovarian cancer, NSCLC }\end{array}$ & $\begin{array}{l}\text { Asymptomatic LVEF } \\
\text { decline [40] }\end{array}$ \\
\hline Dasatinib & TKI & ABL, c-KIT, PDGR & CML, ALL & $\begin{array}{l}\text { QT prolongation, Pericar- } \\
\text { dial effusion }[4,41]\end{array}$ \\
\hline Nilotinib & TKI & ABL, c-KIT, PDGFR & CML, ALL & QT prolongation [41] \\
\hline Sorafenib & TKI & VEGFR, c-KIT & RCC, HCC & $\begin{array}{c}\text { CHF, Acute coronary } \\
\text { syndrome, Hypertension } \\
{[41]}\end{array}$ \\
\hline
\end{tabular}

mAb, monoclonal antibody; CHF, congestive heart failure; LVEF, left ventricular ejection fraction; RCC, renal cell carcinoma; CRC, colorectal cancer; NSCLC, non-small-cell lung cancer; TKI, tyrosine kinase inhibitor; CML, chronic myelogenous leukemia; GIST, gastrointestinal stromal tumor; ALL, acute lymphocytic leukemia; HCC, hepatocellular carcinoma. 